\title{
Communication et coopération dans l'analyse ingénierique
}

\section{Gino Gramaccia}

\section{Q OpenEdition}

1 Journals

Édition électronique

URL : http://journals.openedition.org/communicationorganisation/2216

DOI : 10.4000/communicationorganisation.2216

ISSN : $1775-3546$

Éditeur

Presses universitaires de Bordeaux

Édition imprimée

Date de publication : 1 mai 1999

ISSN : 1168-5549

\section{Référence électronique}

Gino Gramaccia, «Communication et coopération dans l'analyse ingénierique », Communication et organisation [En ligne], 15 | 1999, mis en ligne le 26 mars 2012, consulté le 04 mai 2019. URL : http:// journals.openedition.org/communicationorganisation/2216; DOI : 10.4000/ communicationorganisation.2216

Ce document a été généré automatiquement le 4 mai 2019.

(c) Presses universitaires de Bordeaux 


\title{
Communication et coopération dans l'analyse ingénierique
}

\author{
Gino Gramaccia
}

De nombreux travaux de recherche consacrés, depuis une décennie, à l'étude de nouveaux modèles d'organisation (management par projet, ingénierie concourante, processus transversaux), auront largement développé, dans le champ managérial et à des fins opératoires, deux notions lourdes de présupposés, emportées dans le flot des prescriptions et des connaissances procédurales. Ces notions-clefs sont l'acteur, qu'une forte polysémie a finalement réduit, par commodité, à un sens instrumental, et la coopération, devenue le point d'appui managérial de choix méthodologiques axés, parfois de façon obsessionnelle, sur la quête de performance, de succès productif, ou encore d'« efficience ». Sans doute fallait-il, dans l'esprit de leurs promoteurs, établir et mettre en œuvre un «savoir actionnable» (Argyris, 1995), réinvestir ces deux concepts d'une signification pragmatique, les «instrumentaliser " comme on dit, dans le temps où ils comptent, pourtant, un riche héritage théorique (Weber, Boudon, Crozier et Friedberg). Jean-Claude Moisdon (1992), Philippe Zarifian (1995), Christophe Midler (1996), pour ne citer que quelques auteurs, ont dessiné les contours d'une problématique centrée sur l'optimisation des savoirs et des savoir-faire collectifs dans laquelle la communication est employée comme mode de gestion d'incidents, une technique de régulation des conflits et de recherche de compromis. L'acteur intervient dans le contexte d'une solidarité de crise; il communique au sein d'équipes fébriles, inventant «en temps réel» une rationalité de l'urgence. L'équipe serait-elle, pour reprendre une formule de Midler, une « machine à communiquer » imposant à ses acteurs, à travers l'usage métaphorique que pourraient en faire des ingénieurs organisationnels ${ }^{1}$ les contraintes logiques d'un modèle inspiré des sciences de l'ingénieur? La coopération, au sens où l'entendent des chercheurs exerçant, pour la plupart, au sein de laboratoires de grandes écoles, serait-elle l'expression managériale d'une norme morale visible à peine dans les formes modales discrètes d'un discours sociologique empreint de culture technique? Il est sans doute prématuré d'évoquer ici l'émergence d'un quelconque courant. Parlons tout au plus d'un mouvement auquel nous donnerons le nom d'analyse ingénierique, dont nous relevons, dans cette courte étude, les présupposés les plus significatifs. 


\section{Transparence et intercompréhension}

2 L'activité communicationnelle dans l'analyse ingénierique met face à face des acteurs spontanément communicants. Sans doute, élevés à la hauteur de cette essence idéale de toute communication verbale, ces acteurs entrent-ils en communication comme d'autres entrent en religion, dépouillés de leurs rôles sociaux, de leur position hiérarchique, de leurs croyances et de leurs préjugés ${ }^{2}$. Insistant sur la fonction du langage, P. Zarifian, suivant en cela Habermas, précise en quoi « il permet de réguler le monde social, de lui donner une forme, de stabiliser un tant soit peu son existence, d'édicter des règles de vie commune en société $»^{3}$. Si on prolonge ce raisonnement, on conviendra que la communication intersubjective rapproche deux sujets se conformant à une norme de compréhension mutuelle comparable, dit Boudon, critiquant Habermas, à d'autres modèles idéalisés, (celui de la concurrence pure et parfaite par exemple). Boudon n'a pas tort de critiquer le modèle idéal habermassien de la communication pure et parfaite " qui exclut toute forme de manipulation, de coalition et de stratégie, entre des acteurs équi compétents, clairvoyants, spontanément transparents $»^{4}$. Curieusement, Zarifian réprouve le fonctionnalisme moral de Habermas dans le temps où il promeut une vision fonctionnaliste de la communication au service du paradigme de la coopération. « ... Il n'est pas douteux, nous semble-t-il, que le paradigme de la « mise en coopération » gagne $\mathrm{du}$ terrain et devienne un nouveau réfèrent culturel qui se substitue...au paradigme des tâches et des responsabilités " ${ }^{5}$. L'auteur oppose une "version faible de la coopération, fondée pour l'essentiel sur une meilleure coordination des tâches, à une "version forte " enrichie d'intersubjectivité et d'intercompréhension. Garel et Midler, dans leur typologie des savoirs requis dans les processus cognitifs dans la coopération de conception ${ }^{6}$ (voir infra), évoquent ces savoirs relationnels qui «fluidifient» le fonctionnement de l'organisation ${ }^{7}$. Cette hypothèse repose sur la croyance en une solidarité naturelle proche, dans ses origines (le rapprochement est tentant), de l'utopie rousseauiste sur l'origine des langues, traitant d'une voix naturelle, d'une langue inarticulée, de cette «langue pure de toute supplémentaire ", dont parle, commentant Rousseau, Derrida ${ }^{8}$. Sans doute faut-il pour se comprendre, dans cette version de la coopération, concevoir une figure très paradoxale de l'immédiation, une médiation ineffable qui ferait l'économie de ses formes langagières ou d'approches discursives visant à établir les conditions, pourtant difficiles, de l'intercompréhension (la négociation, par exemple), ou selon les termes de Valérie Carayol, de la logique du gré, comme "système de rapport préalable nécessaire à une logique de l'échange basée sur la réciprocité "9. Les «implications subjectives «croisées» » de Zarifian, au cœur de la version forte, sont les effets souhaités de la neume qu'évoque Derrida, pure vocalisation liturgique, chant inarticulé, souffle inspiré par Dieu.

3 Dans ce cadre problématique, Christophe Midler a introduit la thèse originale d'une symbolique des objets comme éléments de motivation des acteurs dans le management de projet. La proximité des acteurs réunis en "plateau» (dont la source rousseauiste pourrait être une juste métaphore) autour d'ébauches du produit en développement (plans, maquettes, prototypes) serait une pratique efficace de résolution de problèmes, en même temps qu'un facteur important d'identification et de cohésion. "[La] communication inter-métiers passe d'abord par un rapprochement physique des différents acteurs impliqués au plus près du «terrain». En amont, on se réunit sur le "plateau projet» du Bureau d'Études, là où sont les plans et les maquettes. Ensuite 
l'équipe projet émigre dans l'usine où se fabriquent les derniers prototypes et se mettent en place les machines $»^{10}$. Remontons l'histoire. Le management d'une équipe de projet reproduirait-il, dans cette symbolique, les méthodes de surveillance des tâches des artisans du XIII ${ }^{\mathrm{e}}$ siècle? Émile Levasseur, dans son archéologie des métiers avant la Révolution, décrit, (chapitre sur le règlement du travail) ${ }^{11}$ [...] cette proximité du regard qui surveille. Dans l'atelier du serrurier et de l'orfèvre, "les artisans étaient tenus d'exercer une surveillance attentive sur leurs ouvriers afin qu'ils ne commissent pas d'erreur dans leur travail... Le maitre n'avait pas le droit de travailler seul à l'écart; il fallait qu'il exerçât son métier au grand jour, à la vue du public pour lequel il n'avait pas de secrets. L'orfèvre et le serrurier étaient obligés d'avoir leur forge dans leur boutique ». Dans la gestion symbolique des projets selon Midler, les objets physiques (le plan, la maquette, le prototype), s'ils sont les éléments qui permettent de codifier et de jalonner le récit du projet, les traces symboliques d'un parcours narratif, sont aussi les marqueurs d'un travail collectif donnés à voir et à surveiller dans un espace social restreint. Certes, la surveillance ici, à l'image de la vigilance de l'orfèvre, n'est pas explicitement associée à l'acte de punir. Cette pratique permet davantage de porter au regard des acteurs en présence les preuves concrètes de la progression de l'œuvre, de donner aux " artisans " engagés dans l'action du projet des assurances de progrès. Le plan, la maquette, le prototype, réducteurs d'incertitude dans le projet, deviennent alors, si l'on veut être précis, l'allégorie d'un succès. Midler suggère d'inventer " des supports nouveaux pour s'assurer que l'échange inter-métiers s'opère sur des objets et à un niveau pertinent $»^{12}$. Michel Greif, analysant le développement de la communication visuelle dans les usines, lui donne raison quand il note que « la difficulté, dans l'usine d'aujourd'hui, n'est pas de communiquer beaucoup, de loin, mais de communiquer bien, de près " (Greif, 1989) ${ }^{13}$ Curieusement, le management de projet s'efforce de reproduire le modèle physique d'une main qui fabrique et d'un regard qui contrôle; de nouer une relation de confiance par le jeu de deux allégories essentielles du travail artisanal: le geste et le regard. L'immédiation, encore.

\section{La tentation communautaire ${ }^{14}$}

Dans les formes modernes du travail, et en particulier donc, dans le monde des projets, la communication a une signification pragmatique et instrumentale, on le sait bien.

5 Technique de management chargée de dissiper l'incertitude de la création dans les projets, elle intervient également dans la prévention et la gestion des conflits. «L'équipe de projet, dit Midler, doit être passionnée et lucide. Il faut organiser la mobilisation, la lucidité et la solidarité... Il s'agit de construire un système social qui incite à la confiance et à la transparence $\aleph^{15}$. Sans contester la légitimité industrielle de tels objectifs, on peut s'interroger sur la nature et le fonctionnement de métalangages et de stratégies invisibles tels qu'ils sont décrits par les théoriciens de l'analyse ingénierique.

6 S'interrogeant sur l'évolution des rapports entre l'individu et le collectif dans l'univers industriel et les nouvelles formes de régulation dans l'organisation productive ${ }^{16}$, Zarifian développe l'hypothèse selon laquelle le succès de l'engagement individuel dans une communauté de travail dépend du caractère « non substituable » de cet engagement et de sa parfaite aptitude integrative. Le groupe doit reconnaître la valeur irremplaçable de l'apport individuel, tandis que l'individu doit admettre la nécessité de s'intégrer « dans une communauté de travail et d'action». «Cette double reconnaissance nous semble être 
par elle-même, dit l'auteur, une norme de l'activité sociale dont la validité doit encore être trouvée et éprouvée de manière intersubjective ${ }^{17}$.

7 Zarifian (1995) prévient l'objection selon laquelle la notion de communauté pourrait bien être une fiction ${ }^{18}$. Soit. On conviendra surtout qu'il s'agit d'un mythe. Sa construction résulte d'un processus conventionnel symbolique dont les visées identitaires ont été, en son temps, analysées par Weber ${ }^{19}$. Que Zarifian critique, à juste titre, la vision fonctionnaliste du rôle du salarié dans l'activité productive ${ }^{20}$ ne change rien à l'affaire : précisément, la communauté, même (et parce qu'elle est) assortie du l'engagement explicite de l'acteur (par exemple dans un projet) constitue un a priori ordonné du monde, un construit mythique, un référentiel narratif qui impose inlassablement, à qui veut bien l'entendre ou en revendiquer l'efficience toute la force pérenne, anhistorique, de son modèle. Zarifian ne dit rien de ce qui constitue pourtant la dimension mythique majeure de la communauté (et, bien sûr, de toutes les notions dérivées : coopération, adhésion, engagement, respect mutuel et, à la suite de Habermas, de l'intersubjectif) : à savoir sa spécificité sémiotique. Les liens contractuels de la communauté sont tissés sur le modèle d'un acteur archétypique, ou si l'on veut reprendre les catégories de la sémiotique narrative, de l'actant Destinateur, prescripteur des missions du Sujet et garant de l'ordre établi ou devant être rétabli ${ }^{21}$. La communauté serait elle-même une figure syncrétique de la structure actantielle du récit mythique, principe fondateur et point de retour et d'achèvement d'un récit universel, déjà à l'œuvre, par exemple, dans l'imagerie symbolique et parareligieuse du compagnonnage. La communauté suppose l'antériorité construite d'un sens donné à l'action, et qui plus est, d'un sens axiologique, celui d'un système de valeurs dont la fonction de socle n'est pas contestée. Notre auteur parle d'une " norme morale " pour désigner, dans le monde professionnel, les nouvelles formes de régulation sociale entre l'individu et le collectif ${ }^{22}$. Faut-il en venir à cette croyance selon laquelle la conduite des acteurs au travail serait l'expression concrète, figurative, particulière, de ce que nous appellerons une norme narrative (le «schéma narratif » des sémioticiens), laquelle conditionne, quoi qu'il en coûte, le succès de l'agir ? Comment expliquer autrement ce présupposé de cohérence qui fonde toute la réflexion de l'auteur, et d'ailleurs l'ensemble problématique de l'analyse ingénierique? La métaphore du récit est finalement explicite: les acteurs au travail, et ceci est particulièrement vrai dans le cas d'un projet, ont pour mission première de se conformer, à travers leurs pratiques professionnelles et quelles que soient leurs compétences, aux prescriptions d'une logique narrative. Ils déclinent la reproduction (ou le recommencement) d'un récit, sous une forme concrète particulière, évidemment, à partir d'un schéma narratif simple, universel, rapprochant dans le même élan téléologique, le prescripteur de leur mission (les dirigeants, le chef de projet), le bénéficiaire (l'entreprise, l'équipe), le sujet-héros de l'action, etc. On nous objectera qu'une telle modélisation est réductrice. Mais notre analyse porte davantage sur une très improbable pratique de la communication entièrement dédiée à la mise en scène d'un destin, à un jeu d'acteurs soumis aux contraintes d'une logique narrative et condamnés à la répétition d'une vérité communautaire et à l'exercice de la transparence et de la sincérité. Aux antipodes de l'analyse stratégique de Crozier et Friedberg, cette modélisation, explicitement à l'œuvre chez Christophe Midler, ramène la communication à une technologie de la régulation sociale, à une pratique symbiotique (l'intercompréhension subjective), à un métalangage (codifié et maîtrisé par qui ?) de la prévention des conflits, et encore une fois, à l'expression très particulière de valeurs humanistes ${ }^{23}$. Dans cet univers sémantiquement clos et codifié, l'acteur (souvent désigné comme concret), est un sujet intégralement 
conscient, disons sans inconscient, ou alors inconscient de la force performative de ses propos, énonçant un dire informatif sans dire qu'il communique, et au final, susceptible de faire l'économie langagière de sa communication puisque réduite à la dimension beaucoup plus pure de l'échange intersubjectif, coopératif et solidaire. Dans cet univers encore, régi par un métalangage symbolique (les formes du succès symbolisé par exemple, dans un projet, par la progression physique et visible de l'objet innovant), toute pratique professionnelle, assortie ou non de son procédé langagier destiné à en faciliter l'exécution, doit faire l'objet d'une information exhaustive, cohérente, transparente, explicite (par exemple dans un rapport ad hoc). Jean-Pierre Boutinet évoque ce «présupposé de la transparence communicationnelle » pour désigner cette croyance en la transmission d'une information univoque et pure de toute interférences. «Une telle présupposition porte, dit-il, sur l'univocité des intentions entre l'émetteur et le récepteur ainsi que sur l'univocité dans l'interprétation du message transmis $»^{24}$. Cette information ne doit pas laisser entendre qu'elle aurait pu ne pas être dite ou transmise, porter la marque d'un conflit, dissimuler des calculs stratégiques, faire croire à la possibilité d'un échec, évoquer des souffrances, faire apparaitre des formes aporétiques, des ruptures logiques, et pire, des apostasies, des démissions, des questionnements. Du sens échappé, en quelque sorte, ce que Roland Barthes appelle le sens obtus, le sens «qui vient en trop », « têtu et fuyant $»^{25}$.

Le renouveau du modèle de la relation « communautaire ", concept hérité de la sociologie classique (Tönnies, Weber) ${ }^{26}$, inspiré par l'analyse ingénierique à travers sa problématique de la coopération, serait-il l'effet d'une métaphorisation douce des systèmes techniques d'ingénieurs? Ces systèmes sont conformes aux hypothèses techniques qui ont orienté leur conception. Leur transparence a été acquise dès lors que leur caractère opératoire a été validé. Il est étonnant que cette implicite pensée technicienne inspire une psychologie qui confond présupposition cognitive et présupposition normative. Nul savoir stable et établi ne peut garantir la validité des échanges communicationnels, et au final leur transparence. La seule chose dont les locuteurs peuvent pragmatiquement être sûrs, c'est du caractère conventionnel et précaire d'un accord qui définit le cadre empirique d'une communauté fondée sur une norme provisoire ${ }^{27}$.

On s'interroge alors sur la nature et l'efficacité d'un métalangage, ou mieux, d'un discours chargé de mettre en action un référentiel mythique implicite identifiant communication, communautarisation (communalisation). Ce référentiel est considéré par les théoriciens de l'analyse ingénierique comme une condition indispensable à la mise en œuvre de dispositifs organisationnels destinés à assurer l'efficience d'un management par projet. Mais il n'est pas discuté. Rien n'est dit de sa substance axiologique qui implique que soit spontanément consentants, motivés et mobilisés des acteurs voués à assurer le succès de leurs missions. $\mathrm{Au}$ fond, les préconisations majeures du courant ingénierique se concentrent sur la conception et l'installation de systèmes de gestion qui visent à renforcer la coopération et l'intégration des acteurs dans des projets ${ }^{28}$. Ces systèmes sont conçus à partir des caractéristiques d'un sujet idéal, ouvert, flexible, créatif, réactif, tonique, synchronisé29, dont les actions sont censées épouser les trajectoires pragmatiques définies par l'organisation. «Il s'agit de faire internaliser... un minimum de conscience stratégique par un nombre suffisant d'acteurs dans l'entreprise notamment quand les changements exigent une mobilisation globale », préconise Daniel Leroy dans sa définition du management par projet ${ }^{30}$. 
10 Donc ces systèmes sont conçus sur le modèle d'un psychisme idéal, en fonction d'une représentation collective abstraite d'une organisation "efficiente", excluant le sujet concret, celui qui pense pour lui-même et dissimule du sens pour les besoins d'actions stratégiques personnelles, ou pire, en perdant du sens, favorise l'émergence d'actions erratiques, comme on dit, en géologie, de ce qui reste après le passage d'un glacier. Voilà qui nous ramène aux rives d'une sociologie d'inspiration durkheimienne ${ }^{31}$.

\section{L'archéologie du projet}

11 Philippe Zarifian développe cette hypothèse originale selon laquelle le projet est producteur d'un sens symbolique qui a le pouvoir de reconstruire, de reconfigurer, selon l'expression de l'auteur, ses matériaux de base et ses objectifs pratiques. «Le projet est intéressant en ce qu'il prend distance avec le «réel » existant, pour le transformer. La production symbolique a cette vertu très forte de pouvoir créer du nouveau, non à partir de rien, mais en reconfigurant symboliquement et intentionnellement la réalité, avant de la modifier matériellement $\|^{32}$. Ce travail symbolique des acteurs de projet (concepteurs imaginatifs, explorateurs de scénarios à moindre risques, expérimentateurs de formes, schématiseurs, planificateurs, estimateurs...) est constitutif de toute approche projective. Comment en irait-il autrement? Dans un projet, les acteurs communiquent beaucoup. Il convient de modéliser le sens des actions à conduire, et au final d'investir autant de sens que d'argent. Mais, stricto sensu, à quel plan sémiotique intervient cette symbolisation? Dans les visées projectives, donc hypothétiques, qui prennent la forme de modèles pour l'action (un planning prévisionnel, par exemple)? Dans les choix de structure pour la coordination des acteurs (un organigramme technique) ? Dans les choix budgétaires ? Les scénarios de risques? L'utilisation programmée des ressources? Ces considérations renvoient davantage aux choix épistémologiques des sciences de la gestion et leurs prescriptions téléologiques ${ }^{33}$. Si l'on souhaite donner à ce processus de symbolisation une acception théorique restreinte, il faut en rechercher les conditions et les modalités d'émergence au plan, plus implicite, des présupposés de la stratégie managériale. Dans une telle problématique, la question du sens est traitée sous l'angle des moyens qui permettront de modéliser la trajectoire et le statut narratifs des acteurs du projet dans le sens, précisément, d'une harmonisation parfaite des rôles, de la mise en place de conditions favorables à des pratiques de types symbiotiques. C'est du moins ce que préconisent nombre d'auteurs de l'analyse ingénierique. On songe (c'est un premier exemple) à cette surprenante métaphore de Jean-Marie Hazebroucq de l'effet surgénérateur: « un projet, dit-il, doit produire globalement plus de richesse qu'il en a reçu, tant au niveau humain, financier que technique, que pour tous les acteurs, internes ou externes, de l'entreprise $»^{34}$. Produire plus de richesse, c'est aussi produire plus de sens, bien sûr, mais aussi, pour les acteurs producteurs, des éléments spéculaires, des références renvoyées en exemples comme images de succès, bref, des matériaux prêts pour le symbole (comme on dit " prêt à l'emploi »).

12 Le management par projet organise, par le regroupement de ses acteurs, le balisage planifié des parcours, l'engagement visible des partenaires, le croisement, la capitalisation et la mobilisation des savoirs d'experts, enfin l'effet surgénérateur, les conditions pratiques d'une archéologie du succès. Une mémoire collective restreinte (il faudrait dire micro-collective, puisque l'équipe est l'entité sociale primordiale dans un projet), fixant ses repères dans la progression, à chaque fois louangée, des performances, 
formerait une référence mythique constamment disponible, stimulante, responsabilisante ${ }^{35}$ et au final source de cohésion pour les acteurs engagés dans l'entrelacs d'un projet. Ce management ne s'inscrit pas dans une logique de répétition, celle des métiers rattachés à une hiérarchie fonctionnelle, et qui peut faire sans gravité l'économie d'un travail de mémoire, mais dans une logique d'événements particulièrement propice à toute forme de transcription narrative. En somme, derrière chaque projet, une histoire particulière est possible, un conte est possible, dont la finalité narrative évidente serait de se prêter à sa propre relecture, donc à l'analyse rétrospective des décisions prises sur le parcours du projet. "L'innovation est le fruit de ruptures », dit Midler, mais, ajoute-t-il, « elle est aussi le fruit de la mémoire... Ce qui est en jeu dans [les] processus de brainstorming, ce n'est pas le retour à l'instinct, à la naïveté, c'est au contraire une plongée sans contrainte dans la mémoire enfouie $»^{36}$.

\section{La communauté comme complément du pouvoir} mobilisation de savoirs professionnels (techniques et évaluatifs). Dans cette problématique, un acteur efficace est un acteur qui maîtrise et assemble les savoirs utiles à l'exercice de sa tâche dans le cadre de contraintes techniques, économiques et temporelles. Les méthodes de l'ingénierie concourante sont conçues pour faciliter l'intégration de savoirs multiples et différents (Midler, Garel, 1995) au moyen de dispositifs organisationnels et contractuels ad hoc. Philippe Lorino a développé l'idée d'une formalisation communicante pour assurer le pilotage transversal de processus ou de projets. "Le pilotage de processus consiste, dit-il, à construire une superstructure de règles destinées à assurer un niveau minimal de contexte partagé $»^{38}$. Il s'agit de construire une "grammaire » destinée, dès lors qu'elle est judicieusement appliquée, à promouvoir la circulation des savoirs dans un contexte de travail transversal. Les exemples de grammaires formalisâtes pour la structuration d'un minimum de contexte partagé (Lorino) sont nombreux :

18 - Une équipe chargée du développement d'un produit nouveau utilisera, parce qu'elles permettent l'interpénétration productive des savoirs, diverses grammaires formalisantes comme l'analyse de la valeur, le déploiement fonctionnel de la qualité, les méthodes de conception modulaire...

19 - L'organisation des acteurs en plateaux doit permettre la convergence des savoirs, tandis que des techniques d'implication des fournisseurs en amont des projets favorisent l'évaluation partagée et anticipée des risques. Il s'agit, en situation de conception, d'optimiser les savoirs disponibles, de nature hétérogène, au moyen de règles d'ajustement entre les acteurs. En découlent l'installation d'interfaces organisationnelles et juridiques, basées, si nécessaire, sur des compromis. 
20 - Dans son analyse des processus de conception, P. Béguin (1997) ${ }^{39}$ définit deux concepts majeurs en ingénierie concourante : l'intégration et la coordination. L'intégration résulte, selon l'auteur, de deux opérations: la synchronisation cognitive et la mise en œuvre de processus d'anticipation de caractéristiques futures de l'objet en conception. Dans la synchronisation cognitive, les partenaires ont en commun le savoir utile qui leur permet de confronter et de valider des hypothèses de conception; la coordination renvoie, dit l'auteur, à des processus généraux du travail collectif.

21 Naturellement, ce type de rationalisation tire sa légitimité de la rentabilisation des savoirs. La réduction maximale de l'incertitude par la «stimulation cognitive» des acteurs sert la régulation économique des projets. La déclinaison des risques, la scénarisation de ce qui est faisable et de ce qui ne l'est pas, les logiques d'anticipation, relèvent d'une logique d'efficience dans laquelle l'acteur, incité à conjuguer ses savoirs, est considéré comme spontanément et potentiellement communiquant.

L'acteur de projet a donc, dans cette problématique, un statut exclusivement cognitif. Il ne sait pas, il apprend, il sait. Ce qu'il sait de la relation inter-acteurs, des éléments inter techniques et inter-organisationnels suffirait à prendre les décisions qui s'imposent pour assurer au système d'acteurs sa parfaite cohésion. Aucune stratégie n'est requise dont il pourrait être l'acteur ou à l'inverse l'objet, pour mener à bien les ajustements, ou au pire, les compromis nécessaires. Il lui suffit de penser la relation, d'investir un savoir spécifique sur cette relation pour mettre en place les conditions d'une coopération efficace.

23 Quant aux savoirs relationnels, au cœur de la compétence collective, ils sont le principe structurant de la coopération dans les processus transversaux. L'analyse ingénierique les conçoit comme les éléments forts d'un objectif d'apprentissage dans les organisations par projet multipliant les interfaces techniques et organisationnels, et dès lors qu'ils sont maîtrisés, partagés et mobilisés, une condition majeure de succès dans la recherche des compromis $^{40}$. Les savoirs relationnels sont nécessaires à une relation efficace avec autrui. Ils « fluidifient le fonctionnement de l'organisation (exemple sur la loyauté des acteurs, sens de la diplomatie) » (Garel, Midler). Un facteur important de cohésion, donc.

Les capacités d'écoute et d'expression, les dispositions à la coopération, définiraient des compétences générales en communication instrumentale dont on n'est pas sûr (la théorie ne le dit pas) qu'elles procèdent d'un apprentissage et/ou d'un vécu organisationnels. Sans doute ce savoir relationnel est-il bien davantage l'expression de qualités personnelles intrinsèques conformes à un code général de cérémonie, une étiquette managériale réglementant les usages communicationnels. Le discours du faire faire, du faire coopérer, si l'on est manager, puise sa force performative de la croyance partagée par tous les acteurs en la préexistence incontournable de ce code. Il serait sans doute plus exact de parler de métalangage : la fonction instrumentale de la communication (elle transmet des messages informatifs) serait administrée alors, dans cette hypohèse, par une sorte de grammaire courtoise (après la grammaire formalisante de Lorino) constituée d'un jeu somme toute rudimentaire de valeurs-types (l'engagement, la diplomatie, etc.) et de modalités linguistiques simples (devoir, pouvoir, vouloir, croire) ${ }^{41}$.

Dans l'échange des savoirs relationnels, la communication courtoise se met au service d'une explicitation de l'usage rationnel qui peut être fait de la relation inter-acteurs. 

patenté (ou, en l'occurrence, le chercheur qui parle en son nom) de son adhésion aux valeurs indiscutées du second niveau de rationalité. Si l'objectif d'un manager est d'induire la cohésion de son équipe, alors on saisit mieux l'intérêt tactique d'une opération qui consiste à donner explicitement aux acteurs de bonnes raisons d'agir dans le temps où la mise en conformité de leurs actions à un profil-type de valeurs est présupposée comme une relation exclusive. Il n'est pas offert à l'acteur de projet d'autre alternative que de se conformer à la logique communautaire comme "complément du pouvoir ", apparemment conçue, selon les ingénieristes, comme la seule logique susceptible de permettre la libre circulation transversale de savoirs utiles au succès des actions procédurales.

31

Les acteurs d'un projet se donnent ainsi les moyens de s'expliquer mutuellement les conditions de succès du projet. Pour peu qu'ils soient intégralement compétents, ces acteurs s'installent alors sur deux niveaux de rationalité : dispositifs physiques et contractuels du projet,

2. une rationalité fondée sur ce que Raymond Boudon appelle, après Weber, un système de croyances normatives ${ }^{42}$.

Dans cette hypothèse, le premier niveau est constitué des connaissances procédurales qui permettent aux acteurs de formaliser des méthodes de pilotage de processus ${ }^{43}$. Le second niveau regroupe les valeurs dont l'évidence est supposée transcender la communauté des acteurs engagés dans un projet. Il fournit l'arrière-plan normatif du premier niveau de rationalité, dans le temps où il est décrit par les ingénieristes comme supposer absorber totalement la conscience d'appartenance à une communauté de travail conçue comme le complément d'un pouvoir « en dernière instance ». La loyauté, l'engagement, le sens de la diplomatie, sont des exemples de valeurs spécifiques qui finalisent une action de type communautaire, mobilisées par les savoirs relationnels. Mais qu'est-ce qu'un pouvoir « en dernière instance " sinon un pouvoir apte à utiliser «les individus et les collectivités comme des ressources et des instruments et non comme des ensembles possédant autonomie de gestion et personnalité collective ${ }^{44}$, donc un pouvoir propre à susciter les croyances normatives et, "en dernière instance ", à établir les normes d'adhésion communautaire $^{45}$ ?

Poser l'exclusivité de processus cognitifs pour expliquer les jeux d'acteurs dans les organisations transversales amène certains chercheurs au seuil d'interrogations sans réponses. Florence Charue-Duboc, dans une étude très pertinente sur la mise en place chez Rhône-Poulenc d'un modèle centré sur les projets ${ }^{46}$, souligne, en conclusion, les freins à la mise en œuvre de nouveaux paradigmes organisationnels, qui impliquent, entre autres, l'instauration de relations transparentes entre les différents métiers. «L'intégration des contributions, dans une logique d'optimisation globale et de réactivité aux aléas, induit, selon l'auteur, dans ce qui constituait par le passé un domaine d'autonomie, ce qui peut désormais être vécu comme des ingérences d'autres métiers » (p. 63). Priorité est donnée, dans ces formes organisationnelles flexibles, à la circulation des informations, à la convergence des savoirs requis, à la coordination des volontés normalisées, à la coopération d'acteurs-types. Mais peut-on raisonnablement limiter l'analyse de ce changement de paradigme à une confrontation conflictuelle de savoirs, à une intrusion mutuelle dans l'«espace cognitif» privé qui ruinerait les efforts du 
management pour une coopération efficace? La légitimité des objectifs managériaux n'est pas en cause, ici. Dans sa rigueur gestionnaire, l'analyse ingénierique réactualise ce mythe pédagogique vieux comme le monde selon lequel la transmission du savoir serait une opération autonome. Croire en la pureté cybernétique de l'apprentissage, a fortiori organisationnel, conduit à de redoutables apories.

Enfin l'analyse ingénierique ne fait pas explicitement la part des choses entre l'étude descriptive des processus et des rôles, imprégnée de la théorie des systèmes, et une conception idéale qu'elle se fait de ces processus et de ces rôles ${ }^{47}$.

L'acteur de projet est-il un élément objectif, identifiable, (et en tant que tel, susceptible d'être organisé en catégories) d'un phénomène socio-industriel ou, dans un langage métaphorique provisoire, un "chevalier blanc de la transversalité » (J.C. Moisdon) ? Cet auteur se pose explicitement la question : «En fait, on peut se demander si l'identification a priori d'acteurs d'interfaces à partir de caractères souhaitables n'est pas une fausse question $»^{48}$. À moins d'accorder aux dispositifs transversaux une force de contingence telle qu'elle permet «l'émergence et la pérennisation d'acteurs d'interface », ou encore la formation progressive d'une compétence collective, transversale, et par voie de conséquence solidaire. Le présupposé central de l'analyse ingénierique est là: la préexistence d'un acteur solidaire, efficient, compétent, élément dynamique d'un système d'action.

\section{Conclusion}

«La communication doit être non seulement aidée, elle doit être aussi parfois forcée. Pour le projet, la «bonne communication » n'est pas l'absence de tension, celle où les conflits sont redoutés et refoulés ", dit Midler ${ }^{49}$. La communication est pensée selon une norme, évidemment réductrice, qui opère, sans discussion critique, un glissement entre la fonction informative de la communication et une valeur de transparence imposée par les rigueurs productives du management ${ }^{50}$. La vie communautaire en projet, selon l'analyse ingénierique, est vouée à la transparence, quoi qu'il en coûte. Cette conception n'échappe pas au paradoxe bien connu du double lien. À ceci s'ajoute l'étonnante préconisation de la présence et de l'urgence comme critères majeurs de l'efficience : présence physique des acteurs sur des "plateaux» engagés dans des processus tendus vers l'incontournable nécessité de réussir dans des temps « compactés » (F. Charue-Duboc); présence cognitive (présence d'esprit, devrions-nous dire), requise par la complexité des tâches et la multiplicité des savoirs en jeu ; présence psychologique soumise au filtrage d'une norme communautaire d'une grande simplicité morale. Cette abnégation porte un nom en pathologie du stress: le présentéisme pathologique, ou l'art de "tenir son poste même quand on est malade $»^{5151}$. On conviendra que la communauté, comme paradigme de l'analyse ingénierique, est une construction non pas fictive, mais, dans la finalité rigoureusement pragmatique qu'elle poursuit, simplement schématisée, ou encore lissée, comme on dit d'un planning de ressources. Pierre Georges, dans sa chronique du journal Le Monde (voir note 46), dit, à propos du travailleur compulsif, que le travail rend fou. La raison égarée est du sens perdu, celui d'un sujet beaucoup plus complexe, beaucoup plus retors, que celui, schématisé, par l'analyse ingénierique.

39. Pascal Béguin, L'activité de travail: facteur d'intégration dans les processus de conception, in: Ingénierie concourante. De ta technique au social, Paris, Economica, 1997, pp. 105-106 


\section{BIBLIOGRAPHIE}

Afitep, Le management de projet, Paris, AFNOR, 1991.

ALTER Norbert, Sociologie de l'entreprise et de l'innovation, P.U.F., Paris, 1996.

BOUDON Raymond, L'idéologie, Fayard 1986.

BOUTAUD Jean-Jacques, «Images de soi et organisation », in : Communication \& Organisation, $\mathrm{n}^{\circ} \mathrm{l}$, mai 1992.

BOUTINET Jean-Pierre, L'immaturité de la vie adulte, Paris, PU.F, 1998.

BOUTINET Jean-Pierre, Anthropologie du projet, Paris, P.U.F, 1996 (4e édition augmentée).

BROSSARD Pascale, Chanchevrier Claude, Leclair Pierre (sous la direction de), Ingénierie concourante. De la technique au social, Economica, Paris, 1997.

CARAYOL Valérie, «Quelques hypothèses à propos de la médiation », in : Communication \& Organisation $\mathrm{n}^{\circ} \mathrm{ll}$, 1er semestre 1998.

CAZAUBON Christian, Gino Gramaccia, Gérard Massard, Management de projet technique. Méthodes et outils, Paris, Ellipses, 1997.

CHANAL Valérie, Lesca Humbert, Martinet Alain-Charles, « Vers une ingénierie en sciences de gestion », in : Revue Française de Gestion.

CHARLOT Bernard, Du rapport au savoir. Eléments pour une théorie, Paris, Anthropos, 1997.

CHARUE-DUBOC Florence, « Maîtrise d'œuvre, maîtrise d'ouvrage. Pour comprendre l'évolution des projets chez Rhône-Poulenc », in : Gérer et Comprendre, Annales des Mines, septembre 1997.

CHARUE-DUBOC Florence (sous la direction de), Des savoirs en action, Paris, L'Harmattan, 1996.

DURKHEIM Emile, De la division du travail social, Paris, Presses Universitaires de France, 1996 (collection Quadrige).

ECOSIF (sous la direction de Vincent Giard et de Christophe Midler), Pilotages de projet. Diversités et convergences. Paris, Economica. 1993.

GAREL Gilles et Midler Christophe, "Concourance, processus cognitifs et régulation économique », in : Revue Française de Gestion, juin 1995.

GIARD Vincent, Gestion de projets, Paris, Economica, 1991.

GIARD Vincent, Midler Christophe, « Management et gestion de projet », article disponible sur Internet : http//panoramix. univ-paris1.fr/GREGOR/96-ll.hrml

HATCHUEL Armand, «Apprentissages collectifs et activités de conception », in : Revue Française de Gestion, juin-juillet-août 1994.

HAZEBROUCQ Jean-Marie, « La nouvelle conception de la performance : être efficace oui, mais aussi efficient », in : Gestion 2000, n² 2, mars-avril 1995.

HERNIAUX Gérard, Organiser la conduite de projet, Paris, INSEP Editions, 1992.

JOLIVET François, «L'entreprise « orientée projet » : l'apprentissage des organisations », in : Gestion 2000, 6-1995. 
JOLIVET François, «L'organisation matricielle en question. Les nouvelles relations métiers/ projet », in : Cahiers du $2^{e}$ Colloque de l'Institut Qualité Management, 20 novembre 1997.

JOLRVET Christian, NAVARRE Christian, « Grands projets, auto-organisation et métarègles : vers de nouvelles formes de management des grands projets », in : Gestion 2000, Management et Prospective, avril 1993.

LEGOFF Jean-Pierre, Le mythe de l'entreprise, Paris, La Découverte, 1992.

LEMOIGNE L., « Epistémologies constructivistes et sciences de l'organisation », in : Epistémologie et sciences de gestion, Paris, Economica, Paris, 1990.

LENOBLE Jacques, Droit et communication, Paris, Ed. du Cerf, 1994.

LEROY Daniel, « Le management par projets : entre mythes et réalités », in : Revue Française de Gestion, janvier-février 1996, nº 107.

LEVASSEUR Emile, Histoire des classes ouvrières et de l'industrie en France avant 1789, Livre III : L'émancipation de la bourgeoisie aux douzième et treizième siècle.

LIVET Pierre, «Les limitations de la communication », in : Les études philosophiques, avrilseptembre 1987/2-3, pp. 255-275.

LORINO Philippe, «Le développement de la valeur par les processus », in : Revue Française de Gestion, juin-juillet-août 1995.

LYOTARD Jean-François, La condition postmoderne, Paris, Editions de Minuit, 1977.

MARCINIAK Rolande, « La gestion des situations conflictuelles dans les projets », in : La Cible n - 44, septembre 1992.

MARCINIAK Rolande, «Les qualités du manager de projet », entretien avec Rolande Marciniak, in : La Cible $\mathrm{n}^{\circ} 72$, avril 1998.

MIDLER Christophe, L'auto qui n'existait pas, Paris, Inter Editions, 1996.

MIDLER Christophe, «Le responsable de projet, portrait d'un rôle d'influence », in : Gestion 2000, vol. 9, n², 1993, pp. 144-145.

MIDLER Christophe, « La gestion de projet, une affaire d'apprentissage collectif », in : L'Expansion Management Review, mai 1995.

MOISDON Jean-Claude, Weil Benoît, «L'invention d'une voiture : un exercice de relations sociales ", in : Gérer et Comprendre, décembre 1992, n² 29.

NAVARRE Christian, « De la bataille pour mieux produire... à la bataille pour mieux concevoir », in : Gestion 2000, 6, décembre 1992.

PIOTET Françoise, Coopération et contrainte. «A propos des modèles d'Aoki », in : Revue Française de Sociologie, octobre-décembre 1992.

SEGRESTIN Denis, « Introduction à une problématique de la modernisation des rapports sociaux ", in : Revue Française de Science politique, $\mathrm{n}^{\circ}$ 4, août 1987, vol. 37.

TOURAINE Alain, Critique de la modernité, Paris, Fayard, 1992. Veltz Pierre, Zarifian Philippe, «Vers de nouveaux modèles d'organisation? » in : Sociologie du travail, XXX 1/93.

VERIN Hélène, Entrepreneurs, entreprise, histoire d'une idée, Paris, P.U.F. 1982.

ZARIFIAN Philippe, «L'incomplétude de l'organisation par projet et le rôle des exploitants dans l'industrie de masse flexible », in : Pilotages de projet et entreprises, ECOSIP, Economica, 1993. 
WEBER Max, Economie et Société, les catégories de la sociologie, Paris, Plon Pocket, 1995.

ZARIFIAN Philippe, Travail et communication, Paris, Presses Universitaires de France, 1996.

ZARIFIAN Philippe, Le travail et l'événement, L'Harmattan, Paris 1995.

Cf. également le dossier du numéro 46 (1999) de la revue Sciences de la société, Presses

Universitaires du Mirail : Organisation et qualité..

\section{NOTES}

1. Formule empruntée à Chanal Valérie, Lesca Humbert, Martinet Alain-Charles, Vers une ingénierie de la recherche en sciences de gestion, in : Revue Française de Gestion, $n^{\circ} 116$, novembredécembre 1997, p. 41.

2. André Gorz, Misères du présent, richesse du possible, Paris, Galilée, 1997, p. 204.

3. Philippe Zarifian, Le travail et levé-nerumi, L'Harmattan, Paris 1995, p.. 225.

4. Raymond Boudon, L'idéologie, Fayard 1986, p. 119. En premier lieu, ce modèle suppose « une compétence égale sur tous les sujets. Ensuite, - il suppose une vitesse infinie de l'acquisition et de la circulation de l'information. En troisième lieu, il suppose, au sein du groupe de communication, une absence de phénomènes de manipulation, de coalition et de stratégie. En quatrième lieu, parce qu'il suppose que tous aient sur tous les sujets des vues et des vœux clairs et distincts ".

5. Philippe Zarifian, op. cit., p. 13.

6. Gilles Garel et Christophe Midler, Concourance, processus cognitifs et régulation économique, in : Revue française de Gestion, juin 1995, p. 93.

7. Id. «Ces savoirs sont mobilisés pour comprendre les autres, s'en faire comprendre et faire des choix dans le cadre du travail collectif ».

8. Jacques Derrida, De la grammatologie. Les Éditions de Minuit, 1967, p. 351 : « Parler avant savoir parler, telle est la limite vers laquelle obstinément Rousseau conduit sa répétition d'origine». Rousseau (cité par Derrida): "Toutes nos langues sont des ouvrages de l'art. On a longtemps cherché s'il y avait une langue naturelle et commune a tous les hommes; sans doute il y en a une ; et c'est celle que les enfants parlent avant de savoir parler. Cette langue n'est pas articulée, mais elle est accentuée, sonore, intelligible ».

9. Valérie Carayol, Quelques hypothèses à propos de la médiation, in: Communication \& Organisation $\mathrm{n}^{\circ} 11,1^{\mathrm{er}}$ semestre 1998, pp. 63-84.

10. Christophe Midler, L'auto qui n'existait pas, Paris, Inter Editions, 1996, p. 70. Voir également Christophe Midler, Le responsable de projet, portrait d'un rôle d'influence, in: Gestion 2000, vol. 9, n 2, 1993, pp. 144-145.

11. Émile Levasseur, Histoire des classes ouvrières et de l'industrie en France avant 1789, Livre in: L'émancipation de la bourgeoisie aux douzième et treizième siècle, p. 319. « Il est ordoné que nul ne pourra ouvrer en chambre reposte en sa meson de tailler ne de drecier nul garnement, s'il ne le let en l'estabile de l'ouvriez desouz, a la veue du peuple ». Règlement de 1293, Depping, p. 413, cité par Levasseur, p. 320, tome 1.

12. L'auto. op. cil., p. 71.

13. Cité par Midler, id. p. 72.

14. Ce titre est emprunté a Françoise Piotet, Projet $n^{\circ} 221$, Printemps 1990

15. Christophe Midler, La gestion de projet, une affaire d'apprentissage collectif, in : L'Expansion Management Review, mai 1995, pp. 74-75.

16. Philippe Zarifian, Le travail et l'événement. L'Harmattan, Paris, 1995.

17. Idem, p. 229. 
18. Ibid. p. 232.

19. Rappelons tout de même les références d'une paire de concepts souvent cités mais qui doivent être soumis régulièrement a la fraîcheur d'une relecture. Cf. donc le chapitre Communalisation et sociation, in Weber Max, Economie et Société, les catégories de la sociologie. Plon Pocket, p. 78.

20. Idem, p. 228.

21. Dans la terminologie de la sémiotique narrative, les actants, au nombre de six (le Destinateur, le Destinataire, le Sujet, l'objet, l'Adjuvant et l'Opposant), sont définis comme des «contenus » progressivement investis de sens au fur et à mesure que s'élabore le récit. Joseph Courtès propose cette comparaison: «Un peu à l'exemple du héros d'un roman, dont le «portrait » s'élabore au fur et a mesure du récit et qui n'est totalement constitué qu'au terme de la narration : au début, il n'est qu'un support (désigné souvent par un nom " propre », c'est-àdire sans pratiquement aucun contenu sémantique précis) vide, auquel l'auteur rapporte successivement, au fil du roman, un certain nombre de fonctions (ou actions) et/ou de qualifications par lesquelles seules le héros prend corps et se définit». Introduction a la sémiotique narrative et discursive, Hachette Université, Paris, 1976, p. 62.

22. Ibid p. 229.

23. «La générosité, dit Zarifian, n'est pas une valeur. Elle est une manière d'être actif, d'agir de façon raisonnée selon la nécessité et le désir d'associer individu et communauté ». Il dit encore : «La générosité est la forme éthique de l'agir ensemble dans un univers productif» et ce, citant Spinoza, « au nom de la Raison », op.cit., p. 161.

24. Jean-Pierre Boutinet, L'immaturité de la vie adulte. Presses Universitaires de France, 1998, p. 89.

25. Roland Barthes, L'obvie et l'obtus. Seuil, 1982.

26. Voir Denis Segrestin, Introduction a une problématique de la modernisation des rapports sociaux, in : Revue Française de Science politique, n 4, août 1987, vol. 37.

27. Jacques Lenoble, Droit et communication. Paris, Editions du Cerf, 1994, et Jean-Marc Ferry, in : La modernité en questions, collectif sous la direction de Françoise Gaillard, Jacques Poulain, Richard Schusterman, chez le même éditeur, 1998.

28. Voir par exemple Daniel Leroy, Le management par projets entre mythes et réalités, in : Revue Française de Gestion, janvier-février 1996, n 107, p. 117 L'expérience manageriale des grands projets hydroélectriques du Québec est riche d'exemples de cette « internalisation de conscience stratégique » On pense a ce témoignage de Robert Abdallah, retrouvé sur Internet, vantant les mérites d'une équipe de projet efficace: «Les membres doivent être imbus, convaincus de la compétence de son voisin et le respecter ».

29. Voir par exemple Christian Potié, Le management transfonctionnel, in : Qualittque, $\mathrm{n}^{\circ} 71$, $09 / 1995$.

30. Op.cit., p. 118.

31. Voir Bernard Chariot, Du rapport au savoir. Eléments pour une théorie, Paris, Anthropos, 1997, p. 36 .

32. Philippe Zarifian, L'incomplétude de l'organisation par projet et le rôle des exploitants dans l'industrie de masse flexible, in : Pilotages de projet et entreprises, ECOSIP, Economica, 1993, p. 242.

33. «A l'hypothèse causaliste ou déterministe (correspondance cause-effet) que privilégiait te positivisme, nous pouvons sans nous appauvrir, bien au contraire, substituer l'hypothèse de la correspondance « comportement-téléologie ». J.L. Lemoigne., Epistémologies constructivistes et sciences de l'organisation, in : Epistemologie et sciences de gestion, Paris, Economica, 1990, p. 107.

34. Jean-Marie Hazebroucq, La nouvelle conception de ta performance : être efficace oui, mais aussi efficient, in : Grillon 2000, n², mars-avril 1995, p. 79. 
35. Philippe Lorino, Le développement de la valeur par les processus, in: Revue Française de Gestion, juin-juillet-août 1995, 1.104, p. 70 : «L'enjeu apparaissant au niveau du processus, une culture de responsabilité partagée et de coopération doit émerger »

36. Christophe Midler, op.cit, p. 75.

37. Gilles Garel et Christophe Midler, Concourance, processus cognitifs et régulation économique, in : Revue Française de Gestion, juin 1995.

38. Philippe Lorino, Le déploiement de la valeur par les processus, in : Revue Française de Gestion, $\mathrm{n}^{\circ}$ 104, juin-juillet-août 1995, p. 68.

40. Cette notion n'a pas fait l'objet de développements explicites et convaincants. Pierre Leclair, citant François Régnier (1996), la définit comme une sorte d'aptitude tactique intuitive à entrer en communication avec les autres, une forme spécifique de relation publique, si l'on veut. Dans ce cas, la communication devient cette qualité de cœur que l'honnête homme n'aurait pas désavouée. Elle n'est pas la finalité technique d'un apprentissage, mais une intuition qui échappe aux contingences sociales et culturelles, un effet de la transparence entre les êtres jouant de son caractère ineffable pour mieux transmettre des valeurs de coopération. Cette conception domine toute la pensée ingénierique en confinant la communication a sa fonction classique, dans la philosophie humaniste, de complicité cultivée et courtoise.

41. Voirie dictionnaire de Sémiotique de A.J Greimas et de J. Courtés, Hachette Université, 1979.

42. «Ces croyances |ne] peuvent être dites ru vraies ni fausses, mais [leur] autorité peut être analysée en termes rationnels ", Raymond Boudon, L'idéologie, Fayard, Coll. Essais, 1986, p. 93.

43. Valérie Chanal, Humbert Lesca, Alain-Charles Martinet, Vers une ingénierie en sciences de gestion, in : Revue Française de Gestion, $\mathrm{n}^{\circ}$ 116, p. 44.

44. Alain Touraine, Qu'est-ce que la démocratie? Fayard, 1994, p. 98.

45. Sur ce point, on lira avec profit le chapitre « Coopération des acteurs dans la production de la qualité » dans l'article de Michel Domenc et Hélène Marquié, Les acteurs dans les démarches qualité : soumission ou coproduction? in : Sciences de la société. Presses Universitaires du Mirail, $\mathrm{n}^{\circ}$ 46,1999, pp. 38-40.

46. Florence Charue-Duboc, Maîtrise d'œuvre, maîtrise d'ouvrage. Pour comprendre l'évolution des projets chez Rhône-Poulenc, in: Gérer et Comprendre, Annales des Mmes, septembre 1997, pp. 54-64.

47. Jean-Claude Moisdon, Benoît Weil, L'invention d'une voiture: un exercice de relations sociales, in : Gérer et Comprendre, décembre 1992, n²9, pp. 50-58.

48. Idem, p. 57.

49. Op.cit., p. 72 .

50. Dans un ouvrage déjà ancien (La condition postmoderne, 1977), Jean-François Lyotard a critiqué cette conception binaire de la compétence communicationnelle ramenée à l'alternative traditionnelle d'une parole soit manipulatrice, soit simplement informative : « les messages sont dotés de formes et d'effets tout différents selon qu'ils sont par exemple dénotatifs, prescriptifs, évaluatifs, performatifs, etc. II est certain qu'ils n'agissent pas seulement pour autant qu'ils communiquent de l'information. Les réduire à cette fonction, c'est adopter une perspective qui privilégie indûment le point de vue du système et de son seul intérêt ». Jean-François Lyotard, La condition postmoderne, Editions de Minuit, 1977, p. 32

51. Voir Le Monde du jeudi 8 avril 1998, qui consacre une étude à l'émergence des nouvelles pathologies liées à l'activité professionnelle et au culte de la performance. 


\section{RÉSUMÉS}

Les nouveaux modèles d'organisation du travail (management par projet, ingénierie concourante, processus transversaux) ont fait l'objet d'une littérature théorique et méthodologique foisonnante, principalement axée sur la recherche de principes d'efficience productive. L'auteur admet la légitimité d'un mouvement de pensée qui vise à optimiser les conditions de performances d'acteurs coopératifs et communicants, mais critique les présupposés d'un management fondé sur la croyance selon laquelle des acteurs, spontanément dévoués, doués d'une compétence en communication semble-t-il naturelle, fusionneraient dans l'âme collective d'un projet. Dans ce mouvement, que l'auteur appelle analyse ingénier que, la communication est utilisée, précisément, comme une technique d'optimisation.

New models of work organization (project management, concurrent engineering, transversal processes, etc.) have been the subject of a great many theoretical and methodological studies, mainly as regards research on productive efficiency principles. The author recognizes the legitimacy of a school of thought that emphasizes the need to optimize the performance of people who work together. However, he criticizes the premise of a management style based on the belief that project members, even motivated and gifted at communicating, will naturally blend together into the project's group spirit. In this movement, which the author calls engineering analysis, communication is used, precisely, as an optimization technique.

\section{INDEX}

Mots-clés : communication, coopération, management de projet, analyse ingénierique

\section{AUTEUR}

\section{GINO GRAMACCIA}

Gino Gramaccia est maître de conférences à l'IUT de Bordeaux 1 et membre du GREC/O. Il enseigne la Communication et la Gestion de projet. Il vient de publier aux Éditions Ellipses-Marketing, en collaboration avec Christian Cazaubon et Gérard Massard, un ouvrage de méthodologie sur le management de projet technique. 